\title{
1D Numerical Study of Nonlinear Propagation of Finite Amplitude Waves in Traveling Wave Tubes with Varying Cross Section
}

\author{
Zhineng Zhang and Ling Zheng \\ The State Key Laboratory of Mechanical Transmission, Chongqing University, Chongqing 400044, China. \\ Tingfei Yan and Yao Wu \\ Beijing Institute of Spacecraft Environment Engineering, Beijing 100029, China.
}

\begin{abstract}
(Received 30 November 2018; accepted 2 August 2019)
The nonlinear acoustic problem of a finite amplitude plane wave propagating along the axial direction in a traveling wave tube is studied. Based on the one-dimensional Westervelt equation, a one-dimensional nonlinear wave equation is derived in which the cross section of the traveling wave tube is considered. The two-order finite difference scheme is used to solve the nonlinear wave equation. The nonlinear propagation characteristics of a finite amplitude wave in the traveling wave tube is analyzed. In the expanding transition section, the acoustic pressure amplitude of the acoustic wave decreases with the increase of the cross-sectional area of the pipeline. The nonlinear characteristics of the acoustic wave show waveform distortion and harmonic growth. The waveform distortion becomes more serious in the rear of traveling wave tube than in the front of the tube. Considering the acoustic reflection condition at the mouth, the influence of differently shaped diffusion sections on the acoustic pressure distribution in the test section is investigated. The larger the change rate of the diffusion section in an area, the less amplitude of the sound pressure, and the nonlinear effect of the sound wave propagation is weakened. These nonlinear wave propagation characteristics in a travelling wave tube provide important guidance for both designing a uniform sound pressure distribution in the test section and determining the optimal measuring points for different sizes of structures in spacecraft.
\end{abstract}

\section{INTRODUCTION}

The vibration and noise caused by takeoff, inter-stage separation and two ignitions will cause damage to the satellite and its components during the launching process. Therefore, environmental simulation experiments must be carried out underground before launch. A high-intensity acoustic traveling wave tube can be used to both simulate the acoustic environment of the aerospace structure during flight and to check the anti-acoustic fatigue capability of the structure under a strong sound field, which has become an important test method for ground tests. The analysis of the nonlinear propagation law of the finite amplitude wave in the high strength acoustic traveling wave tube can provide a scientific basis for the optimization design of the traveling wave tube structure and the formulation of the noise test method, which is of great significance to the development of the aerospace industry.

Due to the complexity of the nonlinear propagation of the finite amplitude waves in the pipeline, especially for both the complex traveling wave tube structure and its boundary conditions, it is difficult to obtain accurate solutions by the analytical method. ${ }^{1-3}$ Acoustic numerical computation is an effective way to solve the nonlinear propagation problem of finite amplitude waves. At present, there are a large number of documents based on the finite element method, ${ }^{4,5}$ the boundary element method, ${ }^{6}$ and the method of fundamental solutions. ${ }^{7,8}$ These methods are used to calculate the nonlinear sound field in twodimensional and three-dimensional pipeline models. However, the precise model will greatly increase the calculation time. In order to reveal the acoustic propagation law of a simple symmetrical pipe structure, the three-dimensional model can be simplified to a one-dimensional problem. Based on the Kuznetzov wave equation, ${ }^{9}$ T. Tsuchiya used the finite element method to analyse the nonlinear propagation characteristics of finite amplitude waves in one-dimensional straight tubes and two-dimensional exponential tubes, and analyse its frequency-domain response characteristics by Fourier transform as well. ${ }^{10}$ Hallaj used the finite difference time domain method to solve the Westervelt equation and to analyse the problem of nonlinear propagation of finite amplitude waves in an ideal medium. ${ }^{11,12}$ The equation comprehensively considers the nonlinear terms and the dissipative items and can freely select the calculation dimension according to the requirements of the working conditions. The equation is intuitive and simple for performing mathematical modeling. However, the equation cannot consider the influence of pipe section change on the nonlinear acoustic propagation in one dimension. Hou Wei et al. used high-order low-dispersion numerical schemes to solve the one-dimensional finite-amplitude acoustic propagation for a finite exponential horn and analyzed the waveform distortion and the effects of horn's geometry. ${ }^{13}$ The results showed that the sound pressure at the outlet of the horn was affected by the piston's vibration speed, frequency and horn shape. The results also showed that the exponential horn and hyperbolic horn had higher sound source radiation efficiency. 
Based on the characteristics of a finite amplitude wave propagating in the time-space grid in a natural way, the finitedifference time-domain method can be used to gradually calculate to the observation point. ${ }^{14}$ The finite-difference timedomain method can be used to calculate arbitrary waveforms in the time domain and can handle multiple boundary conditions as well as reflections, refractions, transmissions and other acoustic phenomena. This paper is organized simply as follows: the literature is introduced in Section 1. After that, a one-dimensional nonlinear model of finite amplitude plane wave propagating in a traveling wave tube is developed in Section 2 and the finite-difference method is used to solve it. In Section 3, the nonlinear propagation characteristics of the finite amplitude wave in the traveling wave tube are obtained and the influence on the nonlinear acoustic field in differently shaped pipes is discussed. This paper is concluded in Section 4.

\section{NONLINEAR PROPAGATION MODEL OF FINITE AMPLITUDE WAVE}

According to the structure characteristics of a traveling wave tube, a model, as shown in Fig. 1, is established to study the nonlinear propagation characteristics of finite amplitude waves. The pipeline consists of a transition section with a variable section and a test section with a constant section. The length of the transition section is $l_{1}$ and the length of the test section is $l_{2}$. The diffusion section of the traveling wave tube was simplified as an acoustic boundary condition. A coordinate system was set up in the throat of the transition section. The cross section of the pipe along the $x$ axis was set to $S(x)$.The plane-wave source along the $x$-axis direction at the inlet of the pipe $(x=0)$ was given as $p_{\text {source }}=p_{0} \sin \omega t$. $p_{0}$ was the sound pressure amplitude, $\omega$ was the angular frequency, and $t$ was time.

Assuming that the medium was an ideal fluid and the initial state was stationary, the control equation of the finite amplitude wave propagating along the $x$-axis in a variable cross section hard wall pipe was obtained by neglecting the effect of viscosity and heat conduction. ${ }^{15}$

Mass conservation was written as:

$$
S \frac{\partial \rho}{\partial t}+\frac{\partial(S \rho u)}{\partial x}=0 .
$$

Momentum conservation was expressed as follows:

$$
\rho\left(\frac{\partial u}{\partial t}+u \frac{\partial u}{\partial x}\right)+\frac{\partial p}{\partial x}=0 .
$$

Isentropic compression was:

$$
p=p(\rho)
$$

Equations (1)-(3) were expanded and retained to second order to obtain Eqs. (4)-(6):

$$
\begin{aligned}
\frac{\partial \rho^{\prime}}{\partial t}+\rho_{0} \frac{\partial u^{\prime}}{\partial x} & =-\rho^{\prime} \frac{\partial u^{\prime}}{\partial x}-u^{\prime} \frac{\partial \rho^{\prime}}{\partial x}-\frac{\rho_{0} u^{\prime}}{S} \frac{\partial S}{\partial x} ; \\
\frac{\partial p^{\prime}}{\partial x}+\rho_{0} \frac{\partial u^{\prime}}{\partial t} & =-\rho_{0} u^{\prime} \frac{\partial u^{\prime}}{\partial x}-\rho^{\prime} \frac{\partial u^{\prime}}{\partial t} ; \\
\rho^{\prime} & =\frac{1}{c_{0}^{2}} p^{\prime}-\frac{1}{\rho_{0} c_{0}^{4}} \frac{B}{2 A} p^{\prime 2} ;
\end{aligned}
$$

where $p$ was the sound pressure, $\rho$ was the medium density and $u$ was the particle vibration velocity. The corner mark " 0 " indicated the initial state of the fluid medium, which was a function of space and a constant in homogeneous medium. The corner mark " " " indicated the disturbance caused by sound waves, which was a function of space and time. Assuming that the medium was initially stationary, there was $u_{0}=0$, then $u=u^{\prime}$

The relation between sound pressure, time and space was obtained by arranging Eqs. (4)-(6) and eliminating the quantity of density and velocity. Following this, the nonlinear propagation equation of the finite amplitude wave in a variable cross section pipe was obtained:

$$
\frac{\partial^{2} p^{\prime}}{\partial x^{2}}-\frac{1}{c_{0}^{2}} \frac{\partial^{2} p^{\prime}}{\partial t^{2}}+\frac{\beta}{\rho_{0} c_{0}^{4}} \frac{\partial^{2} p^{\prime 2}}{\partial t^{2}}+\frac{\partial p^{\prime}}{\partial x} \frac{\partial(\ln S)}{\partial x}=0 .
$$

In order to simplify the above expression, $p^{\prime}$ was directly recorded as $p . c_{0}$ and $\rho_{0}$ were respectively the propagation velocity of the sound wave and the static density of the medium under the temperature $T=20^{\circ} \mathrm{C}$ and standard atmospheric pressure $P=1.01 \times 10^{5} \mathrm{~Pa}$.

The non-linear coefficient was defined as $\beta=1+B / 2 A$. $B / A$ was called nonlinear parameter ${ }^{16}$ and was expressed as:

$$
\frac{B}{A}=\frac{\rho_{0}}{c_{0}^{2}}\left(\frac{\partial^{2} p}{\partial \rho^{2}}\right)_{s} .
$$

In air medium $B / A=0.4$. All the simulations in this paper were calculated in the air medium under normal temperature and pressure.

Equation (7) was solved using a finite-difference timedomain method. A second order $\left(O\left(\delta_{x}^{2}, \delta_{t}^{2}\right)\right)$ difference scheme as Eq. (9) was constructed, ${ }^{14}$ where $\delta_{x}$ was the spatial step and $\delta_{t}$ was the time step. The discrete representation of the pressure was $p\left(x_{i}, t_{n}\right)$, or simply $p_{t}^{n}$. In the computation domain, the subscript $n$ represented the time and $i$ represented the space

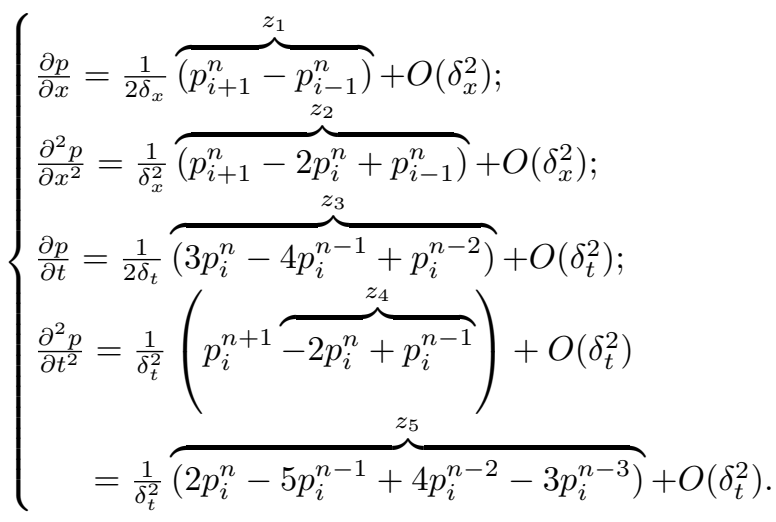

Then, the difference scheme of Eq. (7) was obtained:

$$
\begin{aligned}
p_{i}^{n+1}= & \frac{c_{0}^{2} \delta_{t}^{2}}{\delta_{x}^{2}} z_{2}+\frac{2 \beta}{\rho_{0} c_{0}^{2}}\left(p_{i}^{n} \times z_{5}+\frac{1}{4} z_{3}^{2}\right)+ \\
& \frac{S^{\prime}\left(i \cdot \delta_{x}\right)}{i \cdot \delta_{x}} \frac{c_{0}^{2} \delta_{t}^{2}}{2 \delta_{x}} z_{1}-z_{4} .
\end{aligned}
$$

At the initial moment, if the medium was set to be stationary, the initial condition was:

$$
\left.p\right|_{t=0}=0,\left.\quad \frac{\partial p}{\partial t}\right|_{t=0}=0, \quad x \in[0, L] .
$$




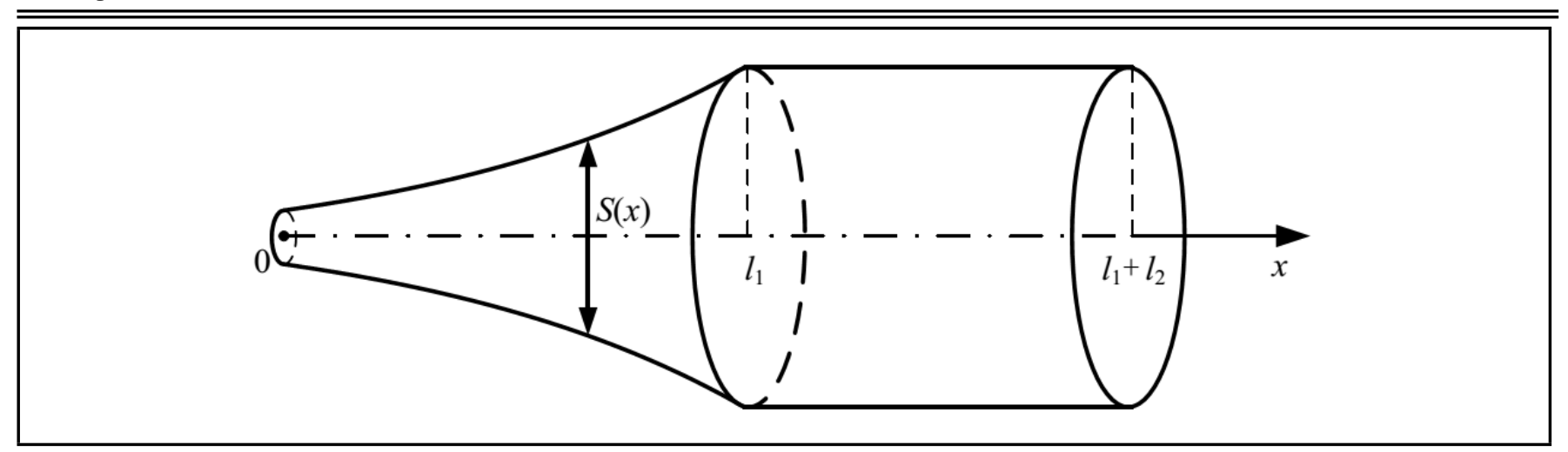

Figure 1. Schematic diagram of a traveling wave tube.

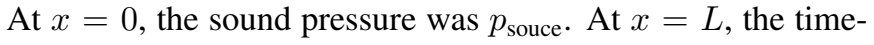
domain impedance boundary condition was taken as boundary condition: ${ }^{17,18}$

$$
\left.p\right|_{x=0}=p_{\text {souce }},\left.\quad\left(\frac{1}{\rho_{0}} \frac{\partial p}{\partial x}+\frac{1}{z_{s}} \frac{\partial p}{\partial t}\right)\right|_{x=L}=0 .
$$

The difference scheme of Eq. (12) was written as:

$$
p_{i+1}^{n}=p_{i-1}^{n}-\frac{\rho_{0} \delta_{x}}{z_{s} \delta_{t}} z_{3} .
$$

Here $L=l_{1}+l_{2}$ represented the range of the computational domain, $z_{s}=\rho_{0} c_{0}\left(\frac{1+R}{1-R}\right)$ represented the medium characteristic impedance, ${ }^{19} R$ was the sound reflection coefficient.

To ensure the stability of the algorithm, considering the $n^{\text {th }}$ harmonic component, the spatial step size was taken as: ${ }^{20}$

$$
\delta_{x}=\frac{c_{0}}{6 n f} .
$$

Here, $f$ was the frequency of sound source.

In general, the relationship between time step and space step was: ${ }^{21}$

$$
\delta_{t} \leq \frac{\delta_{x}}{c_{0} \sqrt{N}}
$$

where $N$ was the spatial dimension of the solution, we took $N=1$. Its physical meaning was that sound waves cannot be transmitted to a space beyond a space step within a time step. For nonlinear equations, simple stability analysis was not applicable. Computational practice showed that it is necessary to determine a relatively conservative time step based on the nonlinear coefficient under the guidance of the above formula, and then multiply this by a stability factor less than 1 to ensure the convergence of the calculation results. ${ }^{22}$ In this paper, the time step was taken as:

$$
\delta_{t}=0.2 \frac{\delta_{x}}{c_{0}}
$$

\section{RESULTS AND ANALYSIS}

\subsection{Model Verification}

To verify the validity of the model represented by Eq. (7), different calculation methods are used to analyse the wave propagation in a traveling wave tube with a constant cross section. These calculation methods took the one-dimensional case of the finite amplitude plane sound wave propagating along the $x$-axis in the ideal medium into consideration. If the sound source is a sinusoidal plane sound wave, the sound wave at the distance from the source $x$ can be expressed by the Fubini analytical solution ${ }^{23}$

$$
u=u_{0} \sum_{n=1}^{\infty} \frac{2 J_{n}(n \sigma)}{n \sigma} \sin [n(\omega t-k x)]
$$

where $\sigma=x / x_{k}, x_{k}=1 /(\beta M a k)$ is the discontinuous distance, $M a=u / c_{0}$ is the sound Mach number, $J_{n}(n \sigma)$ is the $N$-order column Bessel function, $k=\omega / c_{0}$ is the wavenumber. Equation (17) is only applicable to wave propagation before the discontinuity, considering:

$$
u=\frac{p}{\rho_{0} c_{0}} .
$$

Then the relationship between sound pressure and time and propagation distance can be obtained:

$$
p=p_{0} \sum_{n=1}^{\infty} \frac{2 J_{n}(n \sigma)}{n \sigma} \sin [n(\omega t-k x)] .
$$

Assuming the sound source frequency is $f=500 \mathrm{~Hz}$, the sound pressure effective value is $L_{p}=170 \mathrm{~dB}$ (reference sound pressure $P_{\text {ref }}=20 \mu \mathrm{Pa}$ ), the corresponding sound pressure amplitude is $P_{0}=8942.9 \mathrm{~Pa}$. The air density is $\rho_{0}=1.21 \mathrm{~kg} / \mathrm{m}^{3}$, the velocity of $a$ sound wave in the air is $c_{0}=344 \mathrm{~m} / \mathrm{s}$, the nonlinear parameter in the air is $B / A=0.4\left(t=20^{\circ} \mathrm{C}\right)$, the nonlinear coefficient is $\beta=$ $B /(2 A)+1=1.2$, the pipe length is $L=1.4 \mathrm{~m}$, and the pipe section remains unchanged. Take the acoustic boundary conditions at the exit of the pipe as full absorption, i.e. the reflection coefficient $R$ is zero. Figure 2 is a transient time domain waveform under different calculation methods. After degenerating to a straight pipe with equal sections, the nonlinear numerical solution based on Eq. (7) is basically consistent with the transient time waveform of Fubini's analytical solution, which proves that the numerical model can effectively describe the nonlinear propagation of the finite amplitude wave in the traveling wave tube. In addition, during the nonlinear propagation of the finite amplitude wave, the waveform distorts, and the distortion accumulates gradually with the increase of the propagation distance until a discontinuity occurs, and the sawtooth wave is generated. Therefore, Eq. (7) is only applicable to describe waveform propagation before interruption. 


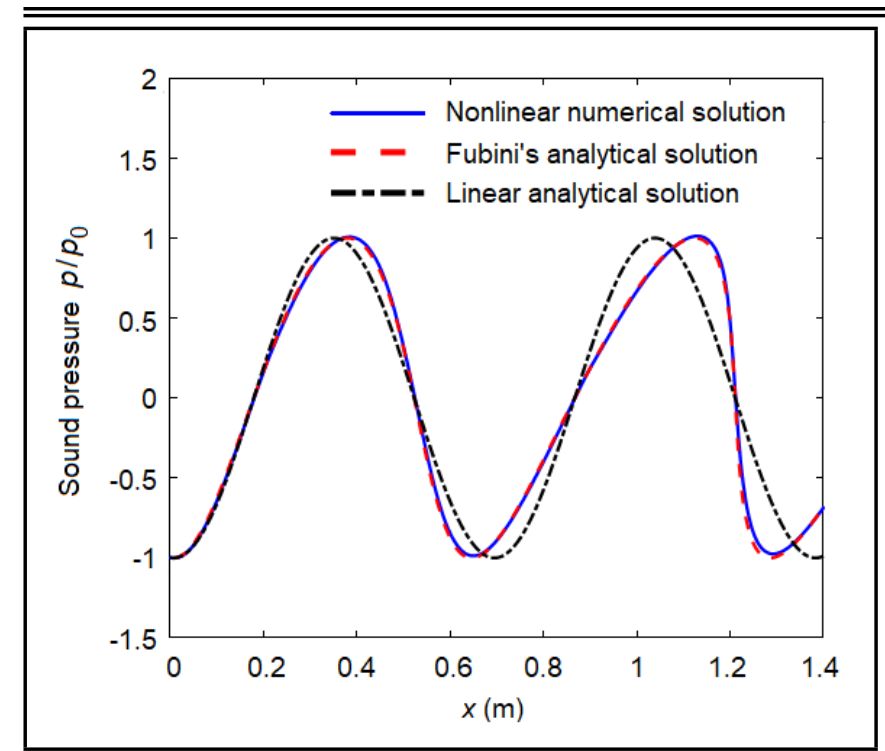

Figure 2. Transient time domain waveform under different calculation methods.

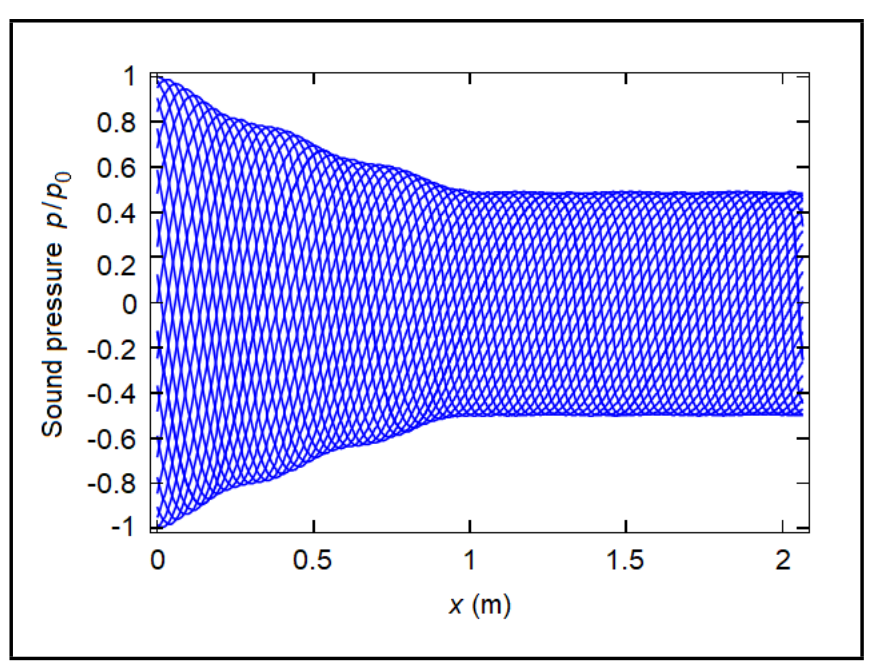

Figure 3. Superposition diagram of time domain waveform at different time $(t=0: 0.004: 0.1 \mathrm{~s})$.

\subsection{Nonlinear Propagation Characteristics in a Traveling Wave Tube}

Assuming the sound source frequency is $f=500 \mathrm{~Hz}$, the sound pressure effective value is $L_{p}=170 \mathrm{~dB}$ (reference sound pressure $P_{\text {ref }}=20 \mu \mathrm{Pa}$ ). The length of the transition section is $l_{1}=1 \mathrm{~m}$, the length of the test section is $l_{2}=1.064 \mathrm{~m}$. The entrance radius of the pipeline is $0.1 \mathrm{~m}$, the exit radius is $0.2 \mathrm{~m}$. The sectional area of an exponential horn is measured by such a law ${ }^{20}$

$$
S(x)=S_{0} e^{\alpha x} ;
$$

where $S_{0}$ is the sectional area of a horn throat, $\alpha$ is the flare constant of the horn. Here take $\alpha=1.3863$. Then, the section area of the pipe can be expressed as

$$
S(x)= \begin{cases}0.0314 e^{1.3863 x}, & 0 \leq x \leq 1 \\ 0.1256, & 1<x \leq 2.064 .\end{cases}
$$

Consider the acoustic boundary conditions at the exit of the pipe as full absorption, i.e. the reflection coefficient $R$ is zero.
Table 1. Spectrum value at different cutoff points.

\begin{tabular}{||c|c|c|c|c|}
\hline Frequency & \multicolumn{4}{|c|}{ Sound pressure (Pa) } \\
\cline { 2 - 5 }$(\mathrm{Hz})$ & $x=1.0 \mathrm{~m}$ & $x=1.3 \mathrm{~m}$ & $x=1.5 \mathrm{~m}$ & $x=1.7 \mathrm{~m}$ \\
\hline 500 & 4292.9 & 4203.4 & 4186.5 & 4117.4 \\
1000 & 1029.2 & 1171.1 & 1271.6 & 1352.2 \\
1500 & 368.8 & 478.2 & 566.4 & 658.6 \\
2000 & 154.8 & 232.6 & 302.1 & 377.5 \\
\hline
\end{tabular}

The nonlinear propagation characteristics of the traveling wave tube are studied in the air medium.

Figure 3 shows a superposition diagram of time domain waveform at different time. In the part of the exponential transition section, the amplitude of the sound pressure decreases with the increase of the section area of the pipe. This is due to the increase of the number of media on the section and the total energy dispersion to more particles, which leads to the decrease of the vibration velocity of the particle and the decrease of the amplitude of the sound pressure. In the test section, the sound pressure amplitude remains unchanged, and the amplitude of the sound pressure amplitude is approximately 0.5 times the sound pressure amplitude of the sound source. The average acoustical power is measured by such a law ${ }^{24}$

$$
\bar{W}=\bar{\varepsilon} c_{0} S=\frac{p^{2} S}{2 \rho_{0}} .
$$

The average sound power of each cross section of pipeline is directly proportional to $p^{2}$ and $S$. The ratio of the cross-section area at the throat of the exponential horn to the straight pipe is $1: 4$ and the ratio of the amplitude of sound pressure is about $2: 1$. The average acoustical power at two places remains unchanged, which shows the conservation of energy and the reliability of the numerical calculation model is verified.

Figure 4 is a waveform diagram of time domain at different cut-off points in the test section. Due to nonlinear effects, the waveform distorts during the propagation process, but the waveform distortion is not the same at different intercept points. The nonlinear effects are cumulative in space, that is, the waveform distortion becomes more serious with the increase of propagation distance.

Table 1 shows the spectrum value at different cutoff points. The high order harmonics will be generated during the nonlinear propagation of the finite amplitude wave. With the increase of the propagation distance from $x=1.0 \mathrm{~m}$ to $x=1.7 \mathrm{~m}$, the fundamental frequency $(500 \mathrm{~Hz})$ energy is continuously transferred to high-frequency $(1000 \mathrm{~Hz}, 1500 \mathrm{~Hz}, 2000 \mathrm{~Hz})$ harmonics, resulting in a decrease in the amplitude of the fundamental frequency and an increase in the amplitude of harmonics.

\subsection{Sound Pressure Distribution in Test Section under Different Shape Transition}

In order to study the effect of different shape transitions on the sound pressure distribution in the test section, four types of variable cross-section ducts, the hyperbolic, conical, exponential, and sinusoidal were used as transition sections. The entrance radius of the four pipeline transition sections is $0.1 \mathrm{~m}$, the exit radius is $0.3 \mathrm{~m}$, the length is $1 \mathrm{~m}$, and the length of the test section is $1.236 \mathrm{~m}$. Table 2 is the schematic diagram of the 


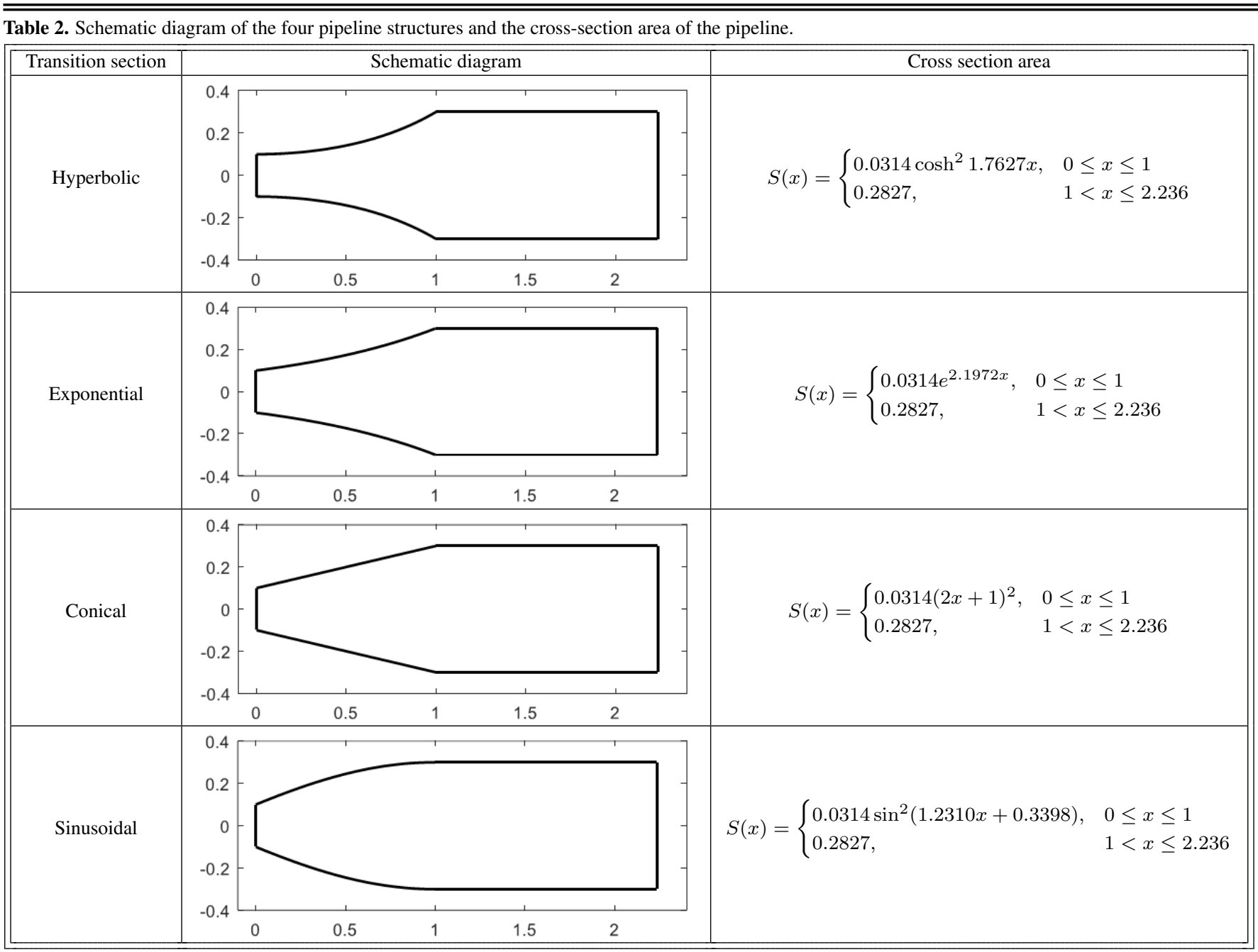

four pipeline structures and the variation rule of the cross section area of the pipeline. According to Eq. (7), when the finite amplitude wave propagates nonlinearly in a cross-section pipe, the influence of pipe cross section is shown in the last item of the equation.

$$
\frac{\partial(\ln S)}{\partial x}=\frac{S^{\prime}(x)}{S(x)} .
$$

$S^{\prime}(x) / S(x)$ is defined as the change rate of the section area of the pipe. Figure 5 shows the change rate of the crosssectional area of the four transitional sections. The crosssectional areas of the four pipelines are gradually increasing, but the change rate is different. The change rate of the cross-section area of the hyperbolic transition section gradually increases, and the exponential transition section remains unchanged. The change rate of the cross-section area of the conical and sinusoidal transition sections gradually decreases, and the sinusoidal reduction rate is faster. The sound source frequency is $f=500 \mathrm{~Hz}$, the sound pressure effective value is $L_{p}=170 \mathrm{~dB}$, the corresponding sound pressure amplitude is $P_{0}=8942.9 \mathrm{~Pa}$. Because the sound pressure at the outlet of the pipeline cannot be fully absorbed during the existing experimental conditions, an impedance boundary condition with a reflection coefficient of $5 \%$ is set at the outlet of the pipeline according to the experimental conditions. The nonlinear propagation of finite vibration waves in the four pipeline models is analyzed.

Figure 6 shows a superposition diagram of time domain waveform of four different pipes. The interaction between the driving sound wave and the reflection wave forms a standing wave in the sound field. The amplitude of the synthetic sound pressure in the space will appear maximum and minimum. In the transition section, the change rate of the sound pressure amplitude with the propagation distance is different due to the different change rate of the cross-section area of the pipeline. The larger the change rate of cross-section area, the faster the amplitude of acoustic pressure decreases. For example, the change rate the cross section area of hyperbolic is from 0 to 3 and sinusoidal is from 7 to 0 , which means that the cross section area of hyperbolic increases slowest and sinusoidal increases fastest at $x=0 \mathrm{~m}$. Accordingly, the amplitude of acoustic pressure of hyperbolic decreases slowest and sinusoidal increases fastest at $x=0 \mathrm{~m}$ from Fig. 6 .

The acoustic waveform diagram of the point $(x=1.5 \mathrm{~m})$ in each pipe is analyzed. As shown in Fig. 7, the distortion of the waveform is different under the action of different transition sections. The waveform distortion is the most serious in the hyperbolic transition section, and the waveform distortion in the sinusoidal transition section is relatively slighter. Figure 8 shows a spectral representation of the harmonics for the four types of cross sections. Table 3 shows the spectral values obtained by the Fourier transform of the time domain waveform. The nonlinear effect of the finite amplitude wave propagating in the pipeline is obvious under the input condition of the sound source. It is shown that the higher order harmon- 


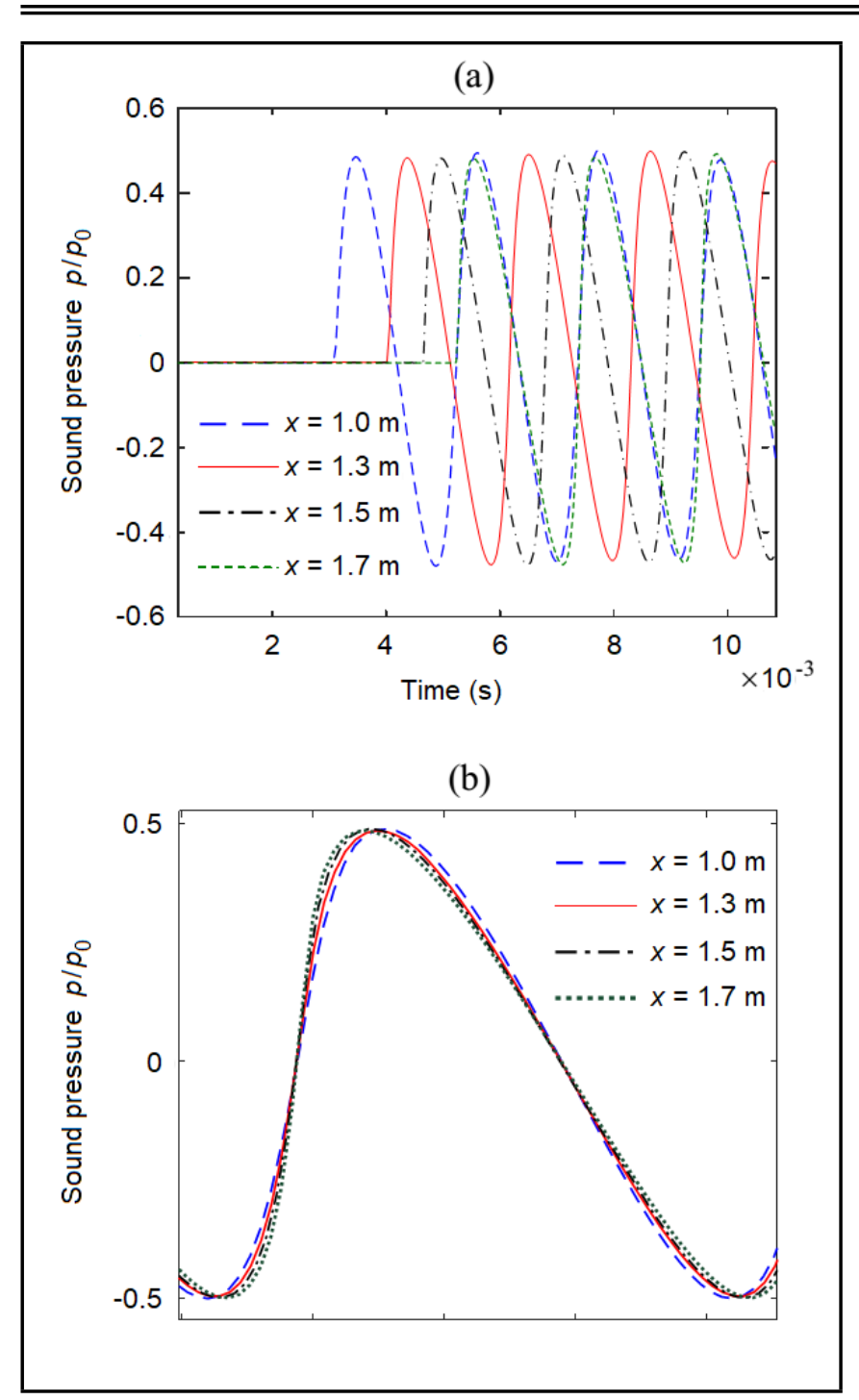

Figure 4. Waveform diagram of time domain. (a) is the waveform diagram of time domain at different cut-off points in the test section, (b) is the waveform diagram of time domain obtained by translating the waveform of (a) to the same starting point.

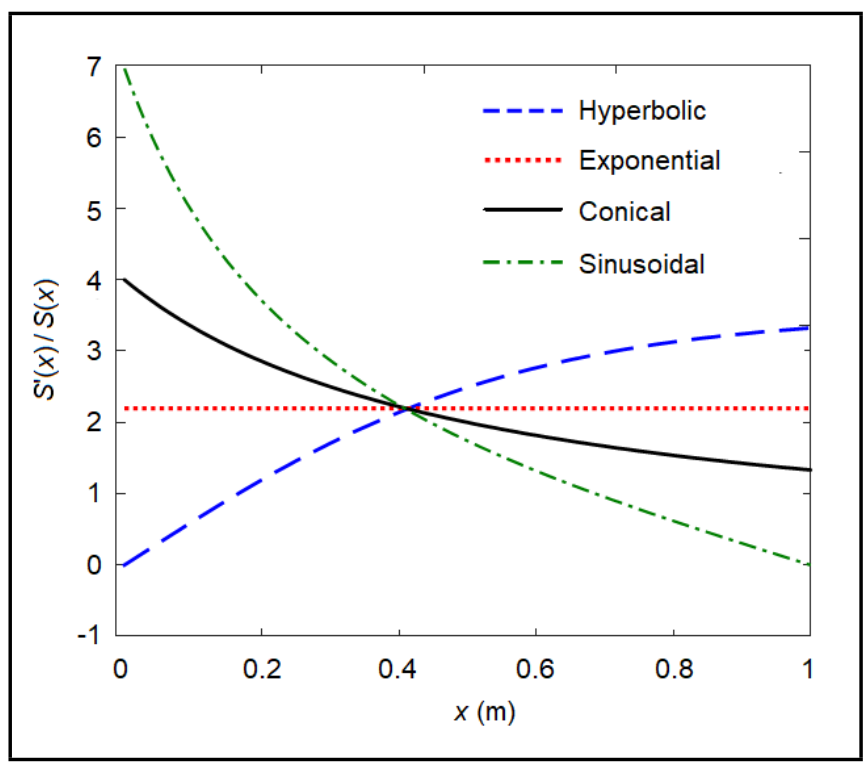

Figure 5. Change rate of the cross-section area of the four transitional sections.
Table 3. Spectral values of the point $(x=1.5 \mathrm{~m})$ in each pipe.

\begin{tabular}{|c|c|c|c|c|}
\hline Transition section & $1^{\text {st }}(\mathrm{dB})$ & $2^{\text {nd }}(\mathrm{dB})$ & $3^{\text {rd }}(\mathrm{dB})$ & $4^{\text {th }}(\mathrm{dB})$ \\
\hline Hyperbolic & 165.1 & 155.0 & 148.9 & 143.6 \\
Exponential & 165.4 & 154.5 & 147.7 & 141.5 \\
Conical & 165.4 & 153.7 & 145.6 & 138.8 \\
Sinusoidal & 165.5 & 152.8 & 143.7 & 135.9 \\
\hline
\end{tabular}

ics are generated, and the higher order harmonic amplitude is larger. The larger the amplitude of higher-order harmonics, the larger the amount of energy that is transferred from fundamental frequency, and thus the more obvious the non-linear effect is. Comparing the difference between the first order harmonic amplitude value and the second order harmonic amplitude value, the smaller the difference between the first-order harmonic and the second-order harmonic sound pressure level is, the more violent the nonlinear change of sound wave is. The difference in the hyperbolic pipe is the smallest $10.1 \mathrm{~dB}$, which shows that the nonlinear effect is the most obvious. The difference in the exponential pipe is $10.9 \mathrm{~dB}$, and the difference in the conical pipe is $11.7 \mathrm{~dB}$. The difference in the sinusoidal pipe is $12.7 \mathrm{~dB}$, indicating that the nonlinear effect is the weakest. This is because the change rate of the sectional area of the sinusoidal pipe is greatest in the front section of the transition section, and the acoustic pressure amplitude is rapidly reduced, and the nonlinear effect is correspondingly reduced. The nonlinear effect accumulated with the propagation distance is smaller than other pipes.

\section{CONCLUSIONS}

Based on the basic equations of fluid dynamics, a onedimensional nonlinear propagation model in a traveling-wave tube is established, which is discretely solved by finite difference time domain finite method. The nonlinear propagation of finite amplitude plane wave in traveling wave tube is studied. Considering the acoustic reflection at the outlet, the influence of different shape transition section on the nonlinear effect in traveling wave tube is analyzed. Research indicates:

1) In the process of non-linear propagation of plane waves, nonlinear effects such as waveform distortion and harmonic generation will occur, and as the propagation distance increases, the waveform distortion becomes more severe and the amplitude of each order harmonic component increases.

2) The increase of the sectional area of the pipeline will lead to the decrease of the sound pressure amplitude. In addition, the expansion of the pipeline will weaken the nonlinear effects in the propagation process. The faster the expansion near the throat, the weaker the nonlinear effects accumulated in the test section.

Therefore, in a design of travelling wave tube, the reduction of sound pressure level caused by pipeline expansion and the weakening of nonlinear effects should be considered fully.

\section{ACKNOWLEDGEMENTS}

The authors acknowledge the National Natural Science Foundation of China (Grant No. 51875061) and Chongqing Science and Technology Commission under Grants (cstc2019jscx-zdztzxX0032) for financial support. 


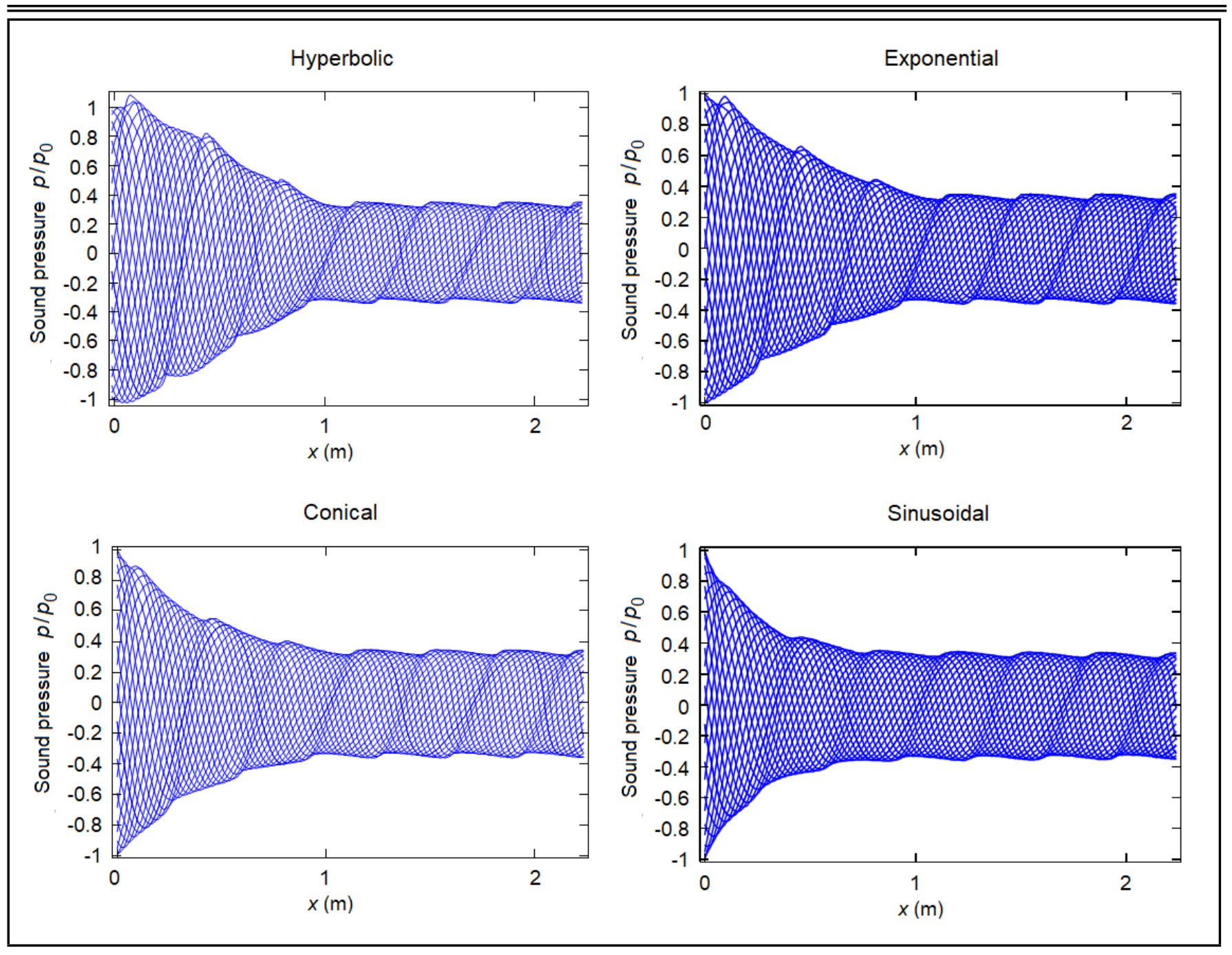

Figure 6. Superposition diagram of time domain waveform of four different pipes ( $t=0: 0.004: 0.1 \mathrm{~s})$.

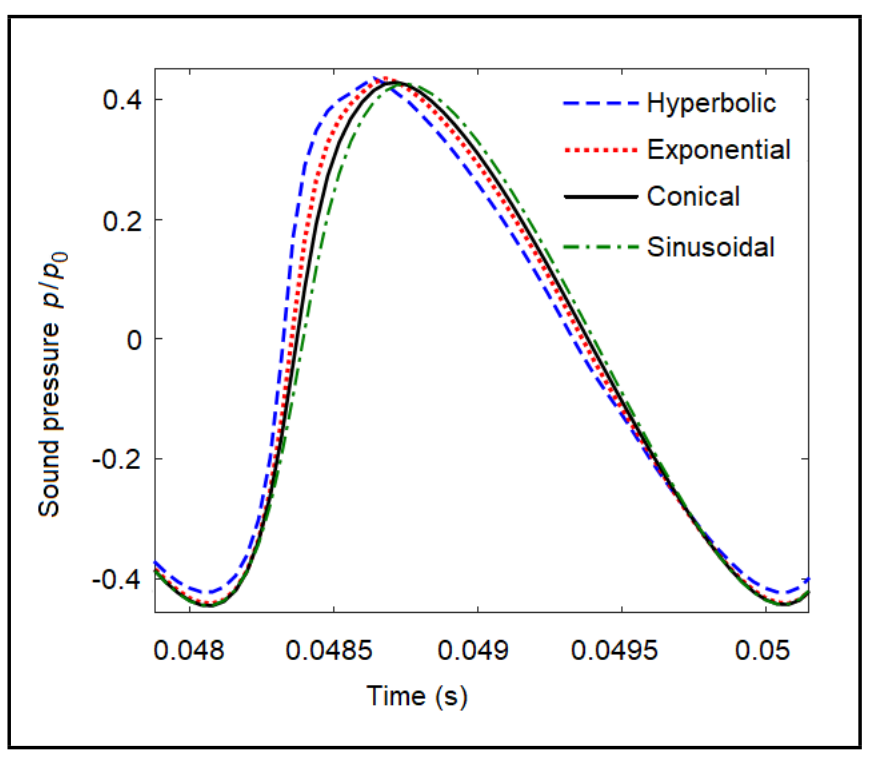

Figure 7. Waveform diagram of the point $(x=1.5 \mathrm{~m})$ in each pipe.

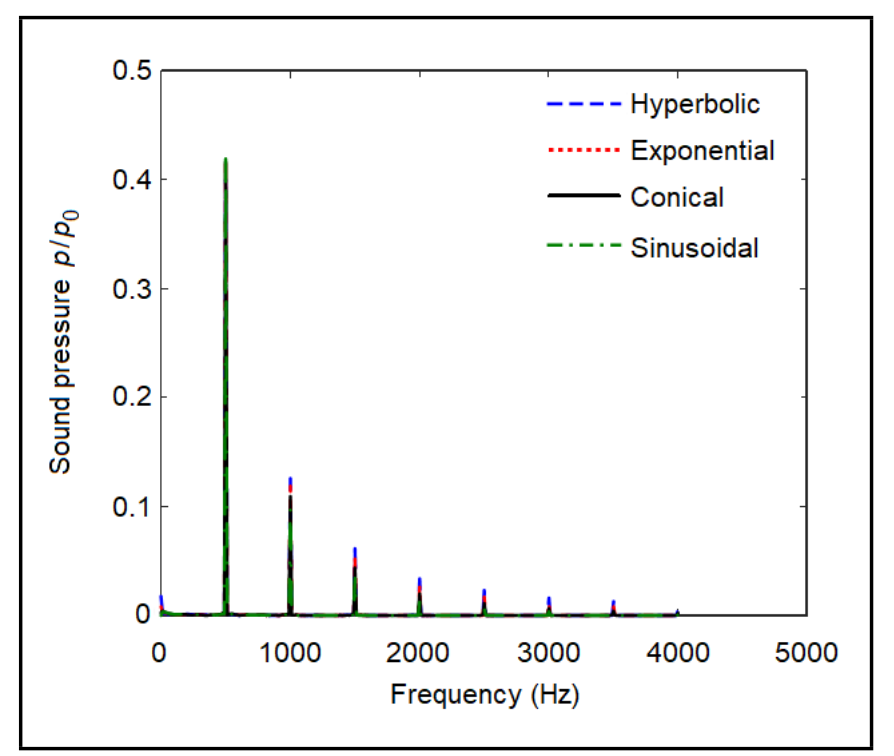

Figure 8. Spectrogram of the point $(x=1.5 \mathrm{~m})$ in each pipe. 


\section{REFERENCES}

1 Webster, A. G. Acoustical impedance and the theory of horns and of the phonograph, Proceedings of the National Academy of Sciences, 5 (7), 275-282, (1919). https://dx.doi.org/10.1073/pnas.5.7.275

2 Goldstein, S. and McLachlan, N. W. Sound waves of finite amplitude in an exponential horn, The Journal of the Acoustical Society of America, 6 (4), 275-278, (1935). https://dx.doi.org/10.1121/1.1915747

3 Kovalev, V. F. and Rudenko, O. V. Nonlinear acoustic waves in channels with variable cross sections, Acoustical Physics, 58 (3), 269-276, (2012). https://dx.doi.org/10.1134/s1063771012030086

4 Barbieri, R. and Barbieri, N. Acoustic horns optimization using finite elements and genetic algorithm, Applied Acoustics, 74 (3), 356-363, (2013). https://dx.doi.org/10.1016/j.apacoust.2012.09.007

5 Vitorino, C. J. T., Barbieri, N., Barbieri, R., and de Lima, K. F. Numerical and experimental study of acoustic horns, Proceedings of the 23rd ABCM International Congress of Mechanical Engineering, (2015). https://dx.doi.org/10.20906/cps/cob-2015-1631

6 Ganesh, M. and Morgenstern, C. High-order FEM-BEM computer models for wave propagation in unbounded and heterogeneous media: Application to time-harmonic acoustic horn problem, Journal of Computational and Applied Mathematics, 307, 183-203, (2016). https://dx.doi.org/10.1016/j.cam.2016.02.024

7 Godinho, L., Amado Mendes, P., Ramis, J., Cardenas, W., and Carbajo, J. A numerical MFS model for computational analysis of acoustic horns, Acta Acustica United with Acustica, 98 (6), 916-927, (2012). https://dx.doi.org/10.3813/aaa.918575

8 Godinho, L., Amado-Mendes, P., Carbajo, J., and RamisSoriano, J. 3D numerical modelling of acoustic horns using the method of fundamental solutions, Engineering Analysis with Boundary Elements, 51, 64-73, (2015). https://dx.doi.org/10.1016/j.enganabound.2014.09.013

9 Kuznetsov, V. P. Equation of nonlinear acoustics, Soviet Physics Acoustics, 16 (4), 467-470, (1971).

10 Tsuchiya, T., Kagawa, Y., Doi, M., and Tsuji, T. Finite element simulation of non-linear acoustic generation in a horn loudspeaker, Journal of Sound and Vibration, 266 (5), 993-1008, (2003). https://dx.doi.org/10.1016/s0022460x(02)01407-4

11 Hallaj, I. M. Nonlinear acoustics in underwater and biomedical applications: Array performance degradation and time reversal invariance, $\mathrm{PhD}$ dissertation, University of Washington, (2013).

12 Westervelt, P. J. Parametric acoustic array, The Journal of the Acoustical Society of America, 35 (4), 535-537, (1963). https://dx.doi.org/10.1121/1.1918525
13 Wei, H., Pan, H., and Song, W. Numerical simulation of nonlinear propagation of sound waves in a finite horn, Chinese Journal of Acoustics, 1429 (2), 138-152, (2015). https://dx.doi.org/10.15949/j.cnki.0217-9776.2015.02.004

14 Taflove, A., Hagness, S. C., and Piket-May, M. Computational Electromagnetics: The Finite-Difference Time-Domain Method, The Electrical Engineering Handbook, Academic Press, 629-670, (2005). https://dx.doi.org/10.1016/b978-012170960-0/50046-3

15 Rudenko, O. V. and Soluyan, S. I. Theoretical Foundations of Nonlinear Acoustics, Springer US, (1977).

16 Beyer, R. T. Parameter of nonlinearity in fluids, The Journal of the Acoustical Society of America, 32 (6), 719-721, (1960). https://dx.doi.org/10.1121/1.1908195

17 Mur, G. Absorbing boundary conditions for the finite-difference approximation of the time-domain electromagnetic-field equations, IEEE Transactions on Electromagnetic Compatibility, EMC-23 (4), 377-382, (1981). https://dx.doi.org/10.1109/temc.1981.303970

18 Tam, C. and Auriault, L. Time-domain impedance boundary condition for computational aeroacoustics, Aeroacoustics Conference, (1996). https://dx.doi.org/10.2514/6.19961662

19 Beyer, R. T. Nonlinear acoustics, Physical Acoustics, 2, 231-264, (1965). https://dx.doi.org/10.1016/b978-0-12395662-0.50014-X

20 Sheng, X.-Q., and Song, W. Finite-Difference TimeDomain Method, Essentials of Computational Electromagnetics, John Wiley \& Sons, Singapore, 207-241, (2012). https://dx.doi.org/10.1002/9780470829646.ch4

21 Chen, J. and Wang, J. Weakly conditionally stable finite-difference time-domain method, IET $\mathrm{Mi}$ crowaves, Antennas \& Propagation, 4 (11), 1927, (2010). https://dx.doi.org/10.1049/iet-map.2009.0542

22 Fletcher, C. A. J. Computational Fluid Dynamics: An Introduction, Computational Techniques for Fluid Dynamics Volume I, Springer, Berlin, Heidelberg, 1-16, (1988). https://dx.doi.org/10.1007/978-3-642-97035-1_1

23 Ivanovna Sokol, G. Infrasound propagation of finite amplitude wave in the big size horn, American Journal of Management Science and Engineering, 2 (5), 117-122, (2017). https://dx.doi.org/10.11648/j.ajmse.20170205.15

24 Bell, L. H. and Bell, D. H. Physical Acoustics, Industrial Noise Control, CRC Press, Boca Raton, 2-35, (2017). https://dx.doi.org/10.1201/9780203751008-2 